\title{
New-onset Alice in Wonderland syndrome following brucellosis
}

\author{
Domenico Chirchiglia, Pasquale Chirchiglia, Rosa Marotta \\ Department Of Neurosurgery, University Of Catanzaro, Italy
}

\begin{abstract}
Background. We report a rare association of Alice in Wonderland syndrome and brucellosis. Alice in Wonderland syndrome is a term applied to altered perceptions of the size or shape of own body or other objects. It can occur in a variety of disorders, including migraine, epilepsy, infections, and brain lesions. Brucellosis is a contagious zoonosis caused by infected milk or meat from animals, mainly goats. It causes a fever known as undulant or Malta fever, associated with gastrointestinal symptoms.

Case. A young 16 year-old male manifests Alice in Wonderland syndrome and migraine headache after suffering from brucellosis. Symptoms are represented by distortion of objects, animals, humans, which appear deformed, so called metamorphopsias. This is a strange case in which a somatosensory syndrome appears after suffering from an infectious disease. Conclusions. Mechanisms of this case are unclear, we hypothesize a toxic-infective cause, targeting brain parietal lobe. responsible for somesthesic functions.
\end{abstract}

Keywords: AIWS, brucellosis, parietal lobe

\section{INTRODUCTION}

Brucellosis is a zoonosis caused by ingestion of unpasteurized milk or infected meat from animals such as goats. It causes a fever known as undulant fever, Malta fever, and Mediterranean fever. Brucella species are Gram-negative bacteria. The symptoms associated are muscular pain and night sweat. The duration of the disease can vary from a few weeks to months. Gastrointestinal symptoms occur in $70-80 \%$ of cases and include nausea, vomiting, decreased appetite, weight loss, abdominal pain, constipation, diarrhea, hepatosplenomegaly. The therapy is based on the use of antibiotics for long periods (5). A serious complication is neurobrucellosis, which affects both the brain and the spine. Spondylodiscitis is a typical response to the vertebral localization of the bacterium, which involves the body and the intervertebral disc, causing damage such as bone erosion and disc thinning.

Alice in Wonderland syndrome (AIWS) is a term applied to altered perceptions of the size or shape of own body or other objects. It is a neurological condition that affects human perception of the senses of vision, hearing, touch, sensation. The name refers to Lewis Carrol's children's book $\mathrm{Al}$ ice's Adventures in Wonderland, in which the title character experiences alterations of sensation in which she felt that her body had grown too tall or too small, or parts of her body were changing shape, size, or relationship to the rest of her body. The syndrome was first described in 1952 by Lippman, and given its name by Todd in 1955 (7).

\section{CASE REPORT}

A 16 year -old male had contracted brucellosis. The symptomatology included intermittent fever, around 38 degrees centigrade, mainly afternoon, abdominal pain, diarrhea, muscular and articular pains, night sweats. Laboratory examinations showed leucopenia, increased hepatic enzymes, and Wright's serum-diagnosis (1/320) as well as blood coltures were positive for brucellosis. 
The presence of a single increase in the IgG antibody stock higher than $1: 160$ is a reliable indication of the current infection. Daily intramuscular injections of streptomycin $1 \mathrm{~g}$ for 14 days and oral $100 \mathrm{mg}$ twice daily for 45 days were administered. After healing, the patient began to see deformed objects, animals and humans, smaller or larger than normal. The patient also did not recognize the parts of his body. The episodes lasted a few hours and had a variable frequency. Brain Magnetic Resonance Imaging, ultrasound-Doppler of supra-aortic vessels, electroencephalogram, psychiatric evaluation were normal. Brain MRI showed both T1 and weighted T2 images, absence of brain tissue alterations. The ultrasonography of the supraortic vessels, carotid and vertebral, showed regular blood flow and absence of obstructive plaques. The electroencephalogram showed regular, symmetrical post-rolandic alpha rhythm, reacting to eye opening. Absence of cerebral electrical activity, both slow and punctiform. Activation tests, hyperpnea and intermittent light stimulation did not modify the electroencephalographic examination A migraine headache was beginning to follow the visual and bodily alterations. Treatment with oral paroxetine $20 \mathrm{mg}$ a day was initiated, and after about a month, the young man had a gradual and clear improvement of visual-spatial episodes and migraine headache.

\section{DISCUSSION}

AIWS is a somatosensory aura preceeding migraine headache. Individuals can experience alterations in visual perception, including micropsia (objects appear small), macropsia (objects appear large), teleopsia (objects appear further away), and pelopsia (objects appear closer). In addition to these alterations in perception, they can also experience hallucinations or illusions of expansion, reduction, or distortion of their own body image. These can include microsomatognosia, in which the individual feels that their own body or body parts are shrinking, or macrosomatognosia, in which one feels that their body or body parts are growing taller or larger. These alterations in size, shape, and perception are known as metamorphopsias or Lilliputian hallucinations. AIWS can occur in a variety of disorders, including migraine, epilepsy, infections, and brain lesions (4).
Techniques using technetium during episodes of AIWS have demonstrated decreased cerebral perfusion in the frontal, parietal, temporal, and occipital lobes. Electrical stimulation of the posterior parietal cortex produced disturbances of body image, including the sensation of body length and size distortion. Neuroimaging has shown that temporoparietal-occipital carrefour (TPO-C) is involved in AIWS, whose symptoms depend on an alteration of TPO-C where visual-spatial and somatosensory informations are integrated. Alterations in these brain regions may cause the occurrence of metamorphopsias and disorders of body schema. AIWS could follow the cortical spreading depression, a mechanism of oligoemia, responsible for migraine aura, which would involve the parietal lobe (3).

Brucellosis is a contagious zoonosis caused by milk and meat contaminated by Brucella conveyed by infected animals, mainly goats. Gastrointestinal symptoms are prevalent and include nausea, vomiting, decreased appetite, weight loss, abdominal pain, constipation, diarrhea, hepatosplenomegaly. Ondulating fever characterizes the disease. The localization of Brucella can occur in bones and joints, provoking osteomyelitis or spondylodiscitis. Diagnosis is based on research of the bacterium in blood cultures, antibodies against the agent with Wright serum-diagnosis, radiological vertebral alterations (8). Neurobrucellosis (NB) represents the localization of bacterium in the brain and spine. Brain symptoms comprise disorder affecting urination and defecation by altered sphyncterial control. Involvement of the cranial nerves has been documented in about $71 \%$ of patients. The positivity rate concerning cerebrospinal fluid standard tube agglutination (STA) has been found around 59\% (9). The radiological/MRI findings have been categorized into four types: normal, white matter changes, vascular insult and inflammatory changes (10). Patients are treated with different combinations of rifampicin, doxycycline, ceftriaxone sodium and sulphamethoxazole for a total of six months. Healing concerns $76 \%$ of patients. Autonomic dysfunctions, especially disorders of urination and defecation as well as hearing loss due to vestibulocochlear nerve injury seem to be typical for NB. The incidence of complications may be related to disease course and involvement of the central nervous system (1). Early detection, diagnosis and treatment could decrease complications (6). 


\section{CONCLUSIONS}

What assumptions can be made on this strange association? AIWS can be triggered by infections, particularly viral such as Epstein Barr virus. Cases of bacterial agents such as Brucellae related to AIWS are not reported in the literature. As with viruses, we could hypothesize a toxic-inflammatory

\section{REFERENCES}

1. Al-Sous MW, Bohlega S, Al-Kawi MZ et al. Neurobrucellosis: Clinical and neuroimaging correlation. Am J Neuroradiol. 2004; 25(3):395401.

2. Chirchiglia D, Chirchiglia P, Marotta R. A singular association of migraine with brainstem aura and Alice in Wonderland syndrome. Childs Nerv Syst. 2019 May 2. doi: 10.1007/s00381-019-04170-8. [Epub ahead of print]

3. Chirchiglia Domenico,Serena Lavano, PasqualeChirchiglia. Neurophysiology of a double aura in migraine and Alice in Wonderland syndrome: Is there a link? Neurology, Psychiatry and Brain Research. Volume 32, June 2019, Pages 1-3.

4. Chirchiglia D, Chirchiglia P, Marotta R. De Chirico and Alice in Wonderland Syndrome: When Neurology Creates Art. Front Neurol. 2018 Aug 13;9:553. doi: 10.3389/fneur.2018.00553. eCollection 2018.

5. Franco MP, Mulder M, Gilman RH, Smits HL. Human brucellosis. Lancet Infect Dis. 2007 Dec;7(12):775-86. action caused by the bacterium on the neurons of the occipital and parietal lobes, causing the appearance of the visual and somatosensory symptoms of AIWS and migraine. Then, the fact that AIWS occurs after Brucella disease would eliminate the hypothesis of AIWS as an its complication.

Conflict of interest: none declared Financial support: none declared

6. Hasanjani R, Mohraz M, Hajiahmadi M, Ramzani A, Valayati AA. Efficacy of gentamicin plus doxycycline versus streptomycin plus doxycycline in the treatment of brucellosis in humans. Clin. Infect. Dis. 2006. 42 (8): 1075-1080. .

7. Mastria G, Mancini V, Viganò A, Di Piero V. Alice in Wonderland Syndrome: A Clinical and Pathophysiological Review. Biomed Res Int. 2016; 2016:8243145.

8. Pappas G, Akritidis N, Bosilkovski M et al. Brucellosis. New Engl J Med. 2005; 352(22):2325-2336.

9. Yetkin MA, Bulut $\mathrm{C}$, Erdinc FS et al. Evaluation of the clinical presentations in neurobrucellosis. Int J Infect Dis. 2006; 10(10):446452.

10. Zheng N, Wang W, Zhang JT, Cao Y, Shao L, Jiang JJ, Huang XS, Tian CL, Yu SY. Neurobrucellosis. Int J Neurosci. 2018 Jan; 128(1):55-62. 